\title{
First experience with a new echographic contrast
} agent

\author{
G Cennamo, N Rosa, G F Vallone, F Smaltino
}

Università 'Federico II'

Napoli, Istituto di

Oftalmologia

G Cennamo

N Rosa

Istituto di Scienze

Radiologiche

G F Vallone

F Smaltino

Correspondence to:

Professor Giovanni Cennamo,

Istituto di Oftalmologia,

Università 'Federico II' di

Napoli, Italy.

Accepted for publication

1 July 1994
Napoli, via S Pansini 5, 80131

\begin{abstract}
The intravenous injection of an ultrasound contrast agent can enhance signals from blood flow. Broad toxicological and pharmaceutical studies in animals confirmed the safety and efficacy of an ultrasound contrast agent made of microparticles of galactose with stabilised microbubbles in watery suspension (SH U $508 \mathrm{~A}$ ). In this paper 10 patients with different malignant orbital and ocular tumours have been evaluated with an echo colour Doppler machine before and after the injection of SH U 508 A. An enhancement of the Doppler signals in the lesions in different
\end{abstract}

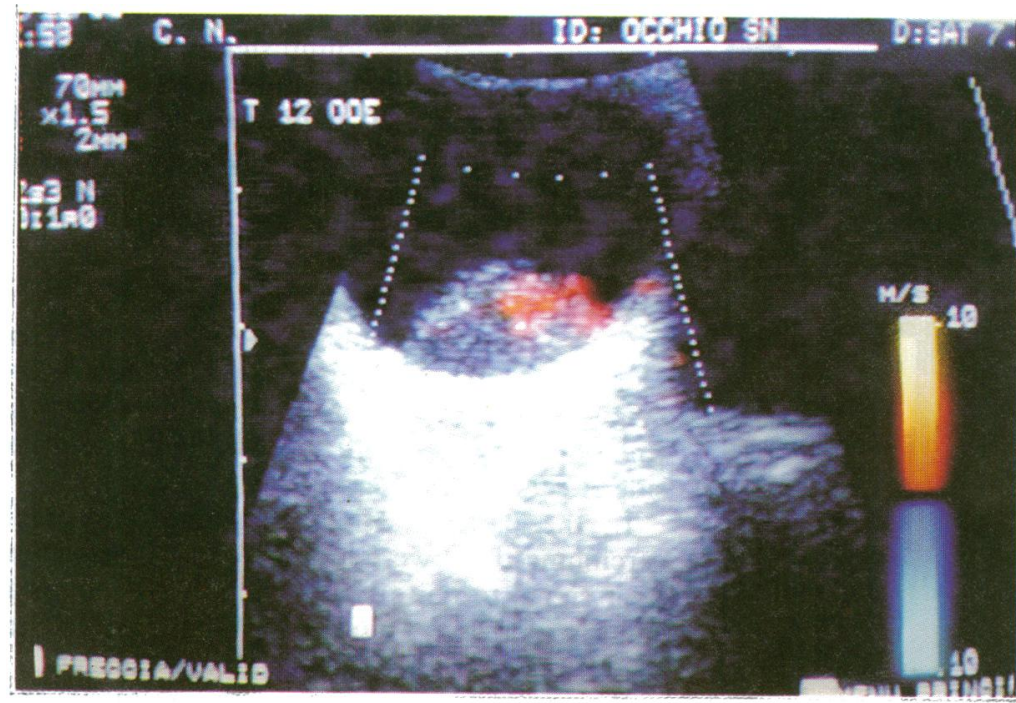

Fig $1 A$

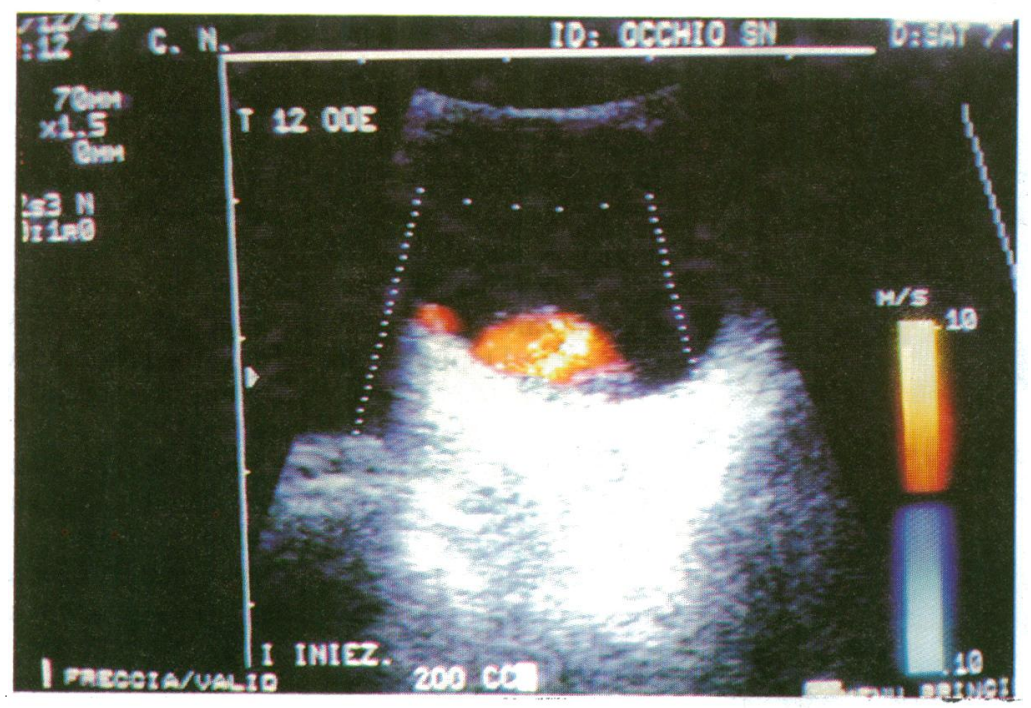

Fig $1 B$

Figure 1 Colour Doppler image of a patient with a large malignant melanoma before $(A)$ and after $(B)$ injection of the contrast agent. A marked enhancement of the signal with the visualisation of new and smaller vessels all over the tumour is present. degrees has been detected. This echographic contrast agent seems to be very important not only in the evaluation of vascular lesions, but also in evaluating the effectiveness of radiotherapy in malignant tumours and could spread the echographic indications in several other ophthalmic fields.

(BrF Ophthalmol 1994; 78: 823-826)

Kinetic echography is one of the criteria in the differential diagnosis of ocular and orbital lesions with standardised echography. ${ }^{12}$ This is a very important criterion in differentiating malignant melanomas from other neoplastic or nonchoroidal lesions. ${ }^{3}$ For this reason a contrast agent that can be injected intravenously, producing an enhancement of the signals from the blood flow, could be very important in the differential diagnosis of several lesions.

With this method flow phenomena can be better seen on echographic scans, the Doppler signal can be enhanced, and quantitative analysis of organic functions may be provided.

According to Ophir and Parker there are five different types of echographic contrast agents: (1) free gas bubbles, (2) encapsulated gas bubbles, (3) colloidal suspensions, (4) emulsions, (5) aqueous solutions.

The action of the contrast agents may be due to three different effects: backscatter; sound attenuation, and changes in the sound speed. However, all of the most promising agents rely entirely upon increased backscatter. The most efficacious are gas bubbles, but their disadvantage is the short shelf life, and inability of these bubbles to survive the pulmonary transit.

A new contrast agent that survives pulmonary transit has been manufactured by Schering (SH U $508 \mathrm{~A}$ ). This agent is made of galactose microparticles which produce microbubbles when mixed with diluent. The bubble size ranges from 2 to $8 \mu \mathrm{m}$ with $97 \%$ of the bubbles less than $6 \mu \mathrm{m} .^{\mathrm{s}}$ Broad toxicological and pharmaceutical studies in animals showed safety and efficacy of this agent. ${ }^{6}$ Studies on a large number of patients confirmed its non-toxicity. ${ }^{78}$

To the best of our knowledge this is the first time that an echographic contrast agent has been used in ophthalmology.

\section{Materials and methods}

Ten patients with different malignant orbital and ocular tumours have been evaluated before and after the injection of SH U $508 \mathrm{~A}$, four were male and six were female, aged ranging from 41 to 80 years. One patient presented with choroidal metastatic carcinoma from breast cancer, six with choroidal malignant melanoma, one patient 


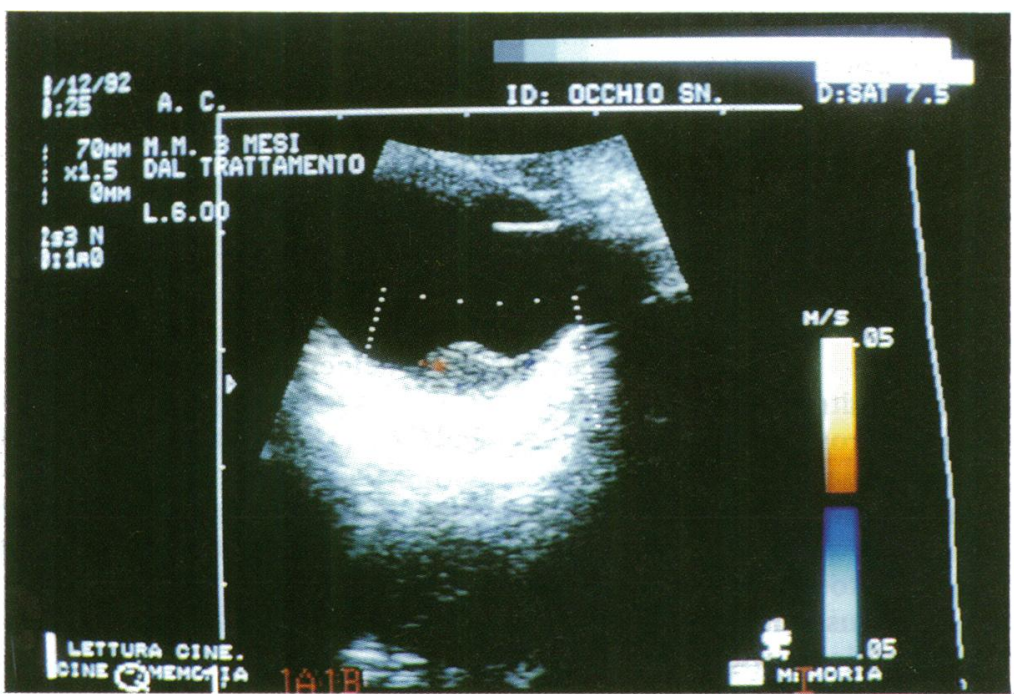

Figure 2 Colour Doppler image of a patient with a large malignant melanoma treated with radiation therapy. The injection of the contrast agent shows no significant Doppler signals.

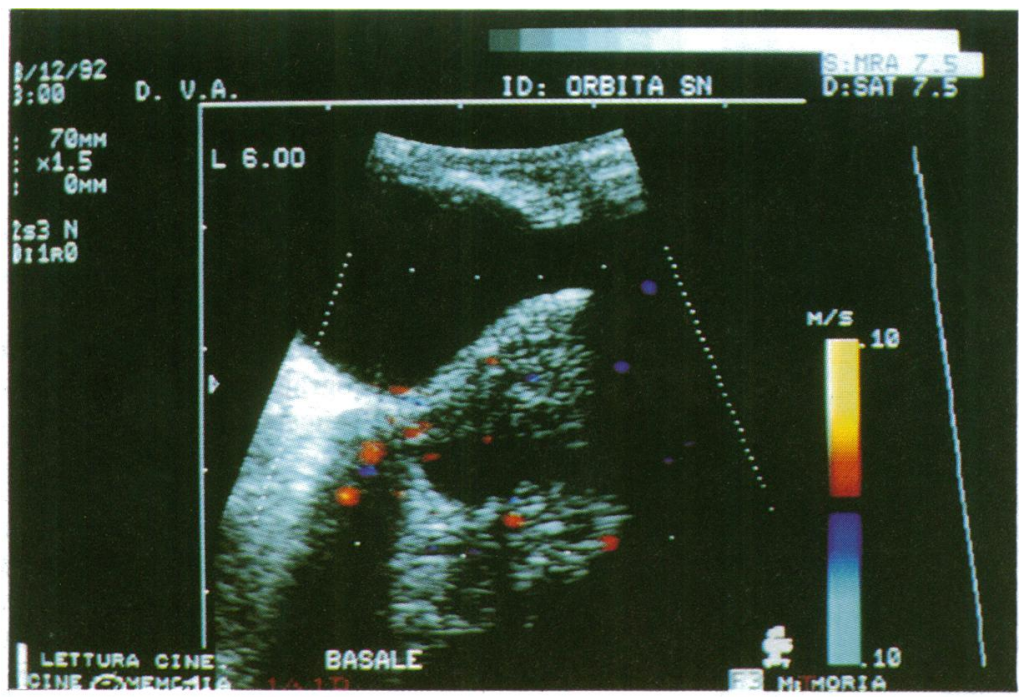

Fig $3 A$

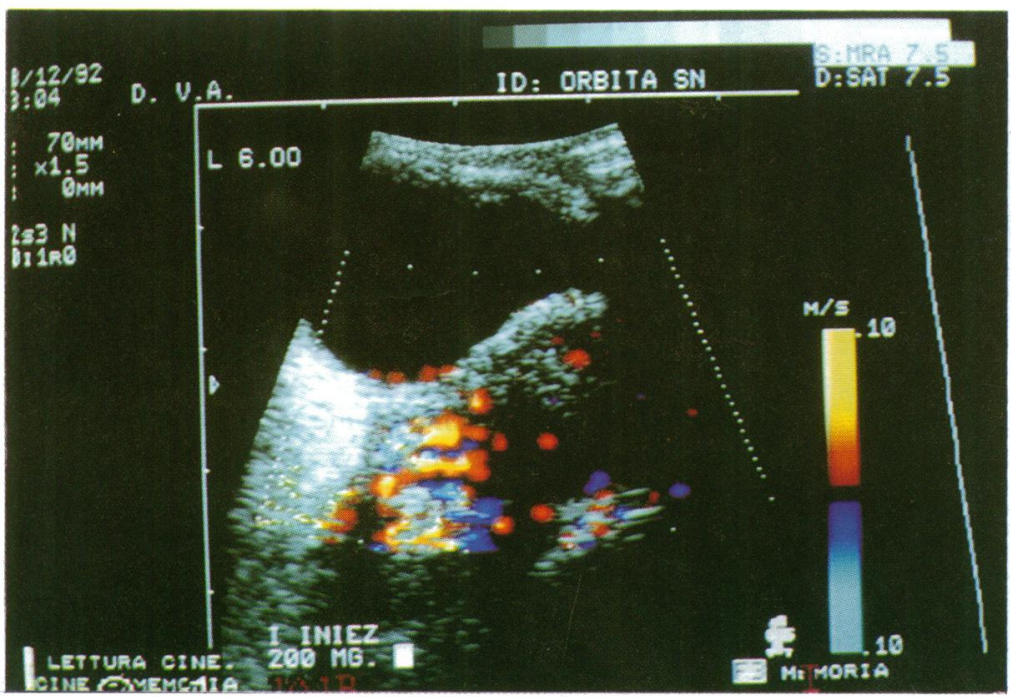

Fig $3 B$

Figure 3 Colour Doppler image of a patient with an orbital carcinoma before $(A)$ and after $(B)$ injection of the contrast agent. A clear cut enhancement of the Doppler signal with the visualisation of new vessels inside the tumour is present. had an orbital lymphoma, and two patients had an orbital metastatic carcinoma. Among the malignant melanomas, four of them were less than $3 \mathrm{~mm}$, two were more than $3 \mathrm{~mm}$, and one of these large malignant melanomas was evaluated 8 months after radiation therapy.

The colour Doppler evaluation was performed after the diagnosis was established on the basis of an echographic examination utilising standardised echographic criteria for the differential diagnosis of orbital and ocular tumours. ${ }^{139}$

The echographic diagnosis was proved in six out of 10 patients by pathology and in the last four cases by the clinical follow up. A biopsy was performed in the two patients with orbital carcinoma, and in the patient with orbital lymphoma. Further, three patients with malignant melanoma (one more and two less than $3 \mathrm{~mm}$ ) were enucleated.

The echo colour Doppler evaluation was performed with a CD Sonochrome by General Electric with a $7.5 \mathrm{MHz}$ probe. During unenhanced baseline scans, pulse repetition frequency (PRF) was set so as to visualise very slow flows. Gains, filters, and colour gating were optimised. The settings were not changed when the contrast agent was administered. It was given by bolus injection into an antecubital vein. The dose of $16 \mathrm{ml}$ at $200 \mathrm{mg} / \mathrm{ml}$ was given to all patients. A $5 \mathrm{ml}$ flush of saline solution followed, to clean the injection cannula.

\section{Results}

In these patients, enhancement of the echo Doppler signal of different degree has been detected, depending on the various lesions. Enhancement lasted 40 to 240 seconds and no side effects were observed.

In the patient with a metastatic choroidal carcinoma there were low Doppler signals with poor signal to noise ratio inside the lesion with slightly increased Doppler signal intensity after the intravenous injection of the contrast agent.

In the four patients with a malignant melanoma of less than $3 \mathrm{~mm}$ there were low Doppler signals with poor signal to noise ratio inside the lesion with no significant changes after the intravenous injection of the contrast agent.

In the patient with a malignant melanoma of more than $3 \mathrm{~mm}$ (Fig 1) there was a good Doppler signal intensity in some areas of the lesion. After the injection there was a marked enhancement of the signal with the visualisation of new and smaller vessels all over the tumour. In the patient who underwent radiation therapy (Fig 2) no Doppler signals from the lesion have been detected, with no significant changes after the injection of the contrast agent.

In the two patients with an orbital carcinoma (Fig ${ }^{\bullet}$ ) there was sufficient Doppler signal intensity before the injection with a clear cut enhancement of the Doppler signal after the injection, and with the visualisation of new and smaller vessels inside the tumour.

In the patient with an orbital lymphoma (Fig 4) there was an almost sufficient Doppler signal intensity inside the lesion, with a moderate enhancement of the Doppler signal after the injection. 


\section{Discussion}

From the early 1970s standardised echography has been used to detect blood flow. With standardised echography the blood flow may be detected either with standardised A scan or Doppler. ${ }^{9}$ In particular in small lesions standardised A scan is more sensitive in detecting blood flow, compared with Doppler, which can detect blood flow only in large vessels. However, the introduction of duplex and colour Doppler ${ }^{10}$ machines allowed a better documentation. ${ }^{11-13}$

For this reason, and for the international trial requirements ${ }^{8}$ that involved specialists in other fields of medicine, we used a colour Doppler to test the efficacy of this new contrast agent. The most promising contrast agents rely entirely upon increased backscatter.

Gramiak and Shah ${ }^{14}$ showed that the agents which most effectively enhance backscatter were those containing free gas bubbles, but these bubbles have two great drawbacks: they are very short lived and are quickly and completely removed by the lungs. Moreover, these bubbles are extremely unstable, and several approaches

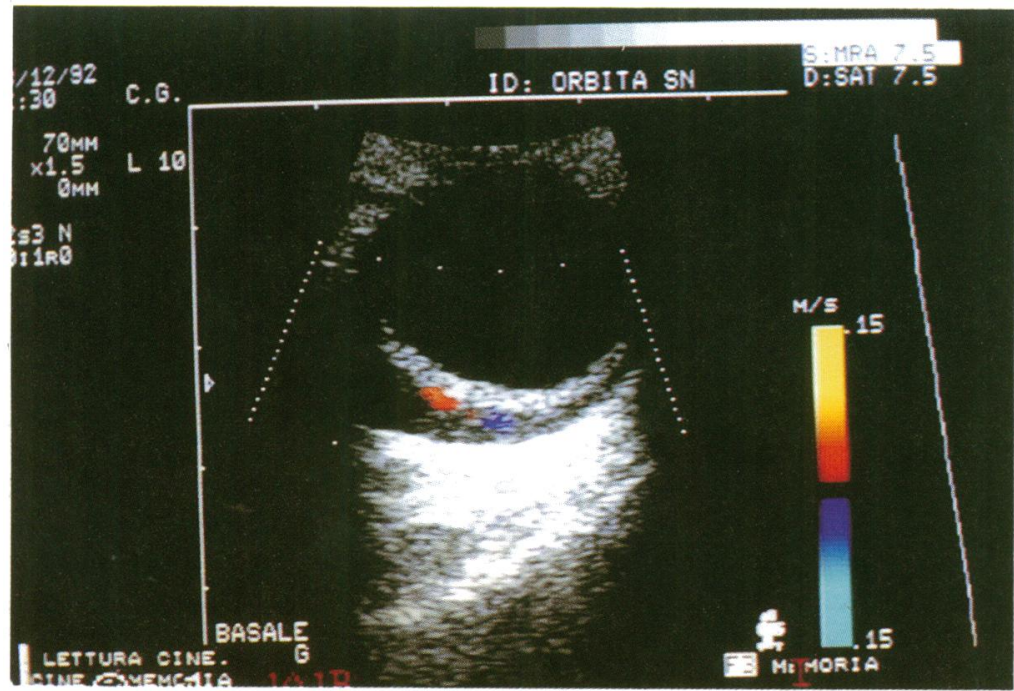

Fig $4 A$

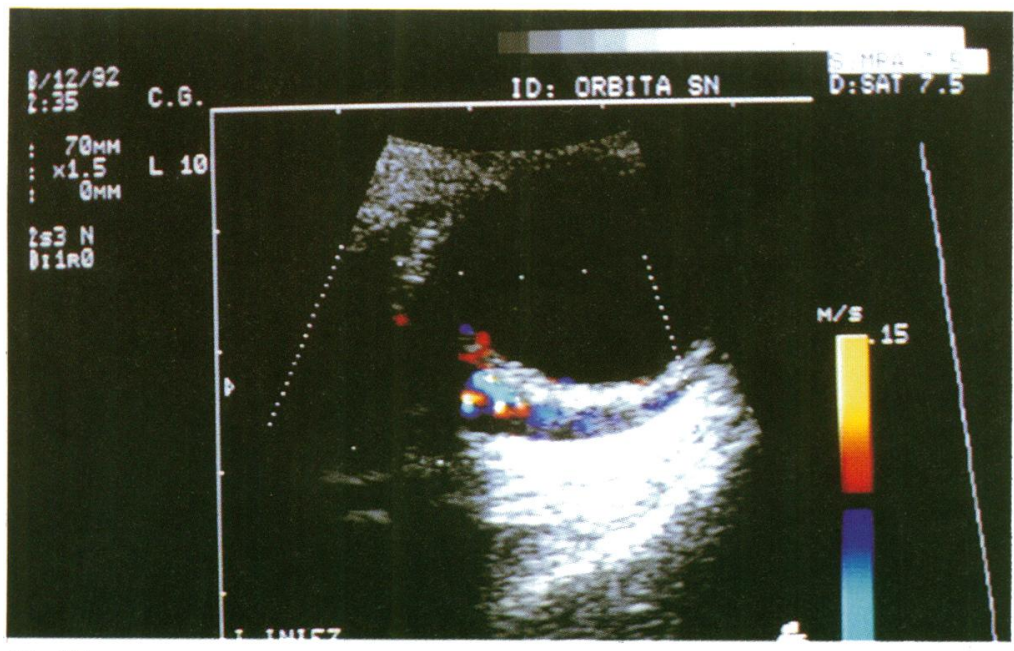

Fig $4 B$

Figure 4 Colour Doppler image of a patient with an orbital lymphoma before $(A)$ and after $(B)$ injection of the contrast agent. A moderate enhancement of the Doppler signal is present. have been adopted to stabilise them - gelatine preparations, viscous solutions, albumin, and saccharides. Albumin and saccharide stabilised solutions are the most promising echo contrast agents currently available. The intravenous injection of a contrast agent that is able to overcome the pulmonary circle may be used to better detect the blood flow in normal and pathological structures. So far the contrast agents available on the market have not been able to survive pulmonary transit, and could not be used in the ophthalmic field.

To survive the lung circle, the agent that we used in this study (SH U $508 \mathrm{~A}$ ), is made of galactose microparticles with an admixture of a very small amount of naturally occurring fatty acid, suspended in water ${ }^{15}$ which produces microbubbles ranging in size from 2 to $8 \mu \mathrm{m}$ with $97 \%$ of them less than $6 \mu \mathrm{m}$.

This size is very important because, to scatter the sound in all directions and not reflect the sound straight back to the source, their diameter should be less than half of the wavelength of the incident sound but not be much smaller than $100 \mu \mathrm{m}$.

On the other hand, to avoid the formation of emboli in the microvasculature and to pass to the lungs, they must be less than $10 \mu \mathrm{m}$ in diameter. Fortunately, when the size of the bubbles falls below $10 \mu \mathrm{m}$, their resonance frequency enters the medical imaging band of $1-10 \mathrm{MHz}$, increasing the scattering effect and overcoming some of the acoustic disadvantage of their small size. ${ }^{16}$

In a previous paper $^{17}$ we proved with an in vitro study that the concentration of the contrast agent used in this clinical trial produces only single spikes from the backscatter, visible with the echographic technique but it does not produce significant changes in the reflectivity and in the sound attenuation inside the lesions.

In conclusion we believe that contrast agents which overcome the lung circle, increasing the signals coming from blood circulation in the vessles, will allow better study of blood flow. This will enable us to evaluate the normal and pathological vascular network in the eye and orbit, and could spread the echographic indications into several other fields of ophthalmology such as the microvasculature of the optic nerve and its head. ${ }^{8}$

1 Frazier Byrne S, Glaser JS. Orbital tissue differentiation with standardized echography. Ophthalmology 1983; 90: 1071 90.

2 Atta HR, Frazier Byrne S. The findings of standardized echography for choroidal folds. Arch Ophthalmol 1988; 106 123441 .

3 Ossoinig KC, Lohmeyer M. Choroidal nevi: diagnosis with standardized echography. Doc Ophthalmol Proc Series 53 1990: 173-80.

4 Ophir J, Parker KJ. Contrast agents in diagnostic ultrasound. Ultrasound Med Biol 1989; 15: 319-33.

5 Schlief R. Ultrasound contrast agent. Curr Opin Radiol 1991; 3: $198-207$.

6 Smith MD, Elion JL, McClure RR, Kwan OL, De Maria AN, Evans J, Fritzsch T. Left heart opacification with peripheral venous injection of a new saccharide echo contrast agent in venous injection of a new saccharide echo
dogs. F Am Coll Cardiol 1989; 13: 1622-8.

7 Schlief R, Schurmann R, Niendorf HP. Blood pool enhancement with SHU508A results of phase II clinical trials. Invest ment with SHU508A results of phas
Radiol 1991; 26 (suppl 1): 188-9.

8 Diagnostic potential of iv contrast enhancement in various areas of cardiovascular Doppler ultrasound - efficacy result of a multinational trial with the galactose-based agent SH $\mathbf{U}$ $508 \mathrm{~A}$. Abstracts of a one day investigators' meeting from 30 January 1993, in Berlin, Germany. Echocardiography 1993 November. 
9 Ossoinig KC. Standardized echography: basic principles, clinical applications and results. Int Ophthalmol Clin 1979, 19: 127-210.

10 Burns PM. Principles of color doppler and colour flow. Radiol Med 1993; 85 (suppl 1): 3-16.

11 Jain R, Sawhey S, Berry M. Real time sonography of orbital tumors. Color doppler characterization: initial experience. tumors. Color doppler characte

12 Guthoff R, Winkler P, Helmke K, Berger R. Diagnosis and treatment control of choroidal melanomas - the role of $\mathrm{B}$ scan and doppler technique. Acta Ophthalmol 1992; 204: $59-61$.

13 Wolff-Korman PG, Kormann BA, Hasenfratz GC, Spengel
FA. Duplex and color doppler ultrasourid in the diagnosis of choroidal tumors. Acta Ophthalmol 1992; 204: 66-70.

14 Gramiak R, Shah PM. Echocardiography of the aortic root. Invest Radiol 1968; 3: 356-66.

15 Fritzsch T, Hilmann J, Kampfe M, Muller N, Schobel C, Siegert J. SH U 508 A a transpulmonary echocontrast agent: initial experience. Invest Radiol 1990; 25: 160-1.

16 Nanda NC. Advances in echocontrast enhancement. Advances in Echo Contrast 1990; 1 : 3-7.

17 Cennamo G, Rosa N, La Rana A, Vallone GF, Smaltino F. Role of the echographic contrast agents in ophthalmology. Doc Ophthalmol Proc Series Proc XV. SIDUO Congress, 1994 (in press). 